\title{
Percutaneous microwave ablation for benign focal liver lesions: Initial clinical results
}

\author{
ZHIGANG CHENG, PING LIANG, XIAOLING YU, ZHIYU HAN, FANGYI LIU, JIE YU and XIN LI \\ Department of Interventional Ultrasound, Chinese People's Liberation Army General Hospital, Beijing 100853, P.R. China
}

Received March 26, 2015; Accepted April 15, 2016

DOI: $10.3892 / \mathrm{ol} .2016 .5409$

\begin{abstract}
Benign focal liver lesions (BFLLs) have become a common disease diagnosed in the clinical setting following the advancement of imaging techniques. The aim of the present study was to evaluate the safety and clinical outcomes of percutaneous microwave (MW) ablation, guided by ultrasound (US), for the treatment of BFLLs. Between May 2005 and January 2011, 44 BFLLs (mean maximum diameter, $33.9 \pm 13.1 \mathrm{~mm}$ ) in 37 patients (23 women and 14 men) were treated by US-guided percutaneous MW ablation with cooled-shaft antennae. The pathological diagnosis of a BFLL was confirmed in 91.9\% (34/37) of patients using a US-guided core needle biopsy prior to ablation. The BFLLs in the other 3 cases were diagnosed by typical presentations on contrast-enhanced imaging, as the patients refused to undergo biopsies. Five inclusion criteria, including indeterminate diagnoses of nodules on contrast-enhanced imaging prior to pathological verification, were recommended. The median follow-up period was 27.0 months (range, 6-73 months). The mean MW ablation energy and time were $55.0 \pm 41.6 \mathrm{~kJ}$ (range, 13.5-207 kJ) and 1,039.9 $\pm 706.2 \mathrm{sec}$ (range, 270-3,450 sec) per lesion, respectively. In total, 40 nodules $(90.9 \%)$ were completely ablated, and no evidence of recurrence was demonstrated on contrast-enhanced imaging follow-up. The other 4 nodules $(9.1 \%)$ were partially ablated due to the proximity of adjacent vital organs. Minor complications without sequelae included local pain and transient hemoglobinuria and no major complications occurred in peri-ablation. Overall, US-guided percutaneous MW ablation is a safe, effective and minimally invasive therapy for BFLLs in selected patients who are non-surgical candidates.
\end{abstract}

Correspondence to: Professor Ping Liang, Department of Interventional Ultrasound, Chinese People's Liberation Army General Hospital, 28 Fuxing Road, Beijing 100853, P.R. China E-mail: liangping301@hotmail.com

Key words: microwave ablation, liver neoplasms, benign, ultrasound guidance

\section{Introduction}

The prevalence of benign focal liver lesions (BFLLs) is high, with up to $52 \%$ of autopsy cases positive for the tumors in the general population (1). With widespread use of ultrasound (US), computed tomography (CT) and magnetic resonance imaging (MRI), $>90 \%$ of BFLLs can be clearly differentiated from liver cancers. Conservative management is recommended for BFLLs such as hepatic cavernous hemangiomas (HCHs) and focal nodular hyperplasias (FNHs) (2), and the use of surgical treatments should mainly be focused on patients with large lesions, symptoms associated with the nodule and lesions with malignant potential. Resection is a common method for treating a BFLL, however, a hepatectomy is a dangerous and complex procedure (3) and indications for the resection of a BFLL must be assessed cautiously (4). More recent non-surgical modalities, such as transcatheter arterial embolization (TAE) (5) and radiofrequency (RF) ablation $(6,7)$, have provided safe, effective and minimally invasive treatments for BFLLs in selected patients.

Microwave (MW) and RF ablation have become increasingly attractive for the treatment of hepatic malignancies (8). Compared with RF, MW energy propagation appears to be less limited by tissue desiccation and charring; thus, MW ablation may destroy a larger lesion than the one destroyed by RF ablation, in a shorter time (9). MW ablation techniques and devices have undergone marked improvements, and MW ablation systems with different frequencies of $2,450 \mathrm{MHz}$ and $915 \mathrm{MHz}$ have been successfully applied in the clinical treatment of hepatocellular carcinoma (HCC) (10). To the best of our knowledge, there have been no published reports concerning US-guided percutaneous MW ablation in the treatment of BFLLs. Therefore, the purpose of the present retrospective study was to evaluate the safety and clinical outcomes of US-guided percutaneous MW ablation with cooled-shaft antennae for the treatment of BFLL in selected patients.

\section{Materials and methods}

Patients. Between May 2005 and January 2011, 37 consecutive patients with 44 BFLLs were referred to the Department of Interventional Ultrasound, Chinese People's Liberation Army General Hospital (Beijing, China) for MW ablation therapy. In total, 31 patients presented with 1 nodule, 5 patients 
with 2 nodules and 1 patient with 3 nodules (Table I). The cohort consisted of 23 women and 14 men, with a mean age of 43.6 \pm 10.6 years (range, $23-70$ years). The diagnosis of BFLL was pathologically confirmed by US-guided core needle biopsy prior to ablation in $34(91.9 \%)$ patients, including 11 patients with $\mathrm{HCH}, 5$ patients with $\mathrm{FNH}, 6$ patients with inflammatory pseudotumors of the liver, 5 patients with solitary necrotic nodules, 3 patients with hepatic adenoma (HA), 3 patients with angiomyolipoma and 1 patient with hepatic epithelioid hemangioendothelioma (HEHE). The diagnosis of $\mathrm{HCH}$ in the other 3 cases $(8.1 \%)$ was confirmed by typical presentations on contrast-enhanced CT or MRI, as the patients refused to undergo biopsies. The second and the third liver nodules in 1 patient were determined according to an identical presentation compared with the first nodule on contrast-enhanced imaging. The mean maximum diameter of the 44 nodules was 33.0 $\pm 19.1 \mathrm{~mm}$ (range, 8-98 mm).

Inclusion criteria for the study were as follows: i) Difficulty in forming a determinate diagnosis for the hepatic neoplasm on the basis of the presentation on contrast-enhanced imaging, particularly in those patients with chronic infection of hepatitis B or $\mathrm{C}$ virus, liver cirrhosis or a history of malignancy. ii) A markedly enlarged BFLL with a maximum diameter that had increased $>1 \mathrm{~cm}$ within 1 year, as determined by routine observation. iii) The neoplasm had malignant potential. iv) Symptoms, such as local pain, discomfort or compression, were considered to be associated with the liver nodule. v) Evident psychological pressure for the detection of the hepatic neoplasm, despite a definite diagnosis of a benign lesion on imaging. In the present study, all patients matched one or more of these five inclusion criteria. Exclusion criteria included: i) Severe blood coagulation dysfunction (a prothrombin time of $>30 \mathrm{sec}$, prothrombin activity of $<40 \%$ and a platelet count of $<30 \times 10^{9} / 1$ cells), ii) acute or active inflammatory and infectious lesions in any organ; and iii) acute or severe renal failure, pulmonary insufficiency or heart dysfunction. This clinical study was approved by the Institutional Review Board of the Chinese People's Liberation Army General Hospital. Written informed consent was obtained from all patients prior to US-guided biopsy and MW ablation.

Pre-ablation imaging work-up. All patients received US, contrast-enhanced US, and contrast-enhanced CT or gadolinium-enhanced MRI to characterize the target nodules prior to ablation. US and contrast-enhanced US were performed using the Sequoia 512 system (Acuson; Siemens AG, Munich, Germany) with 3.5- to 5.0-MHz multifrequency transducers. The US contrast agent was Sonovue (Bracco, Milano, Italy). All CT studies were performed with the same multi-detector row CT machine (Lightspeed 16; GE Healthcare, Wauwatosa, WI, USA) and iopromide contrast medium ( $1 \mathrm{ml} / \mathrm{kg}$ body weight; Ultravist 300; Schering AG, Berlin, Germany). All MRI studies were performed with the same 1.5-T system (Signa Echo-Speed; GE Healthcare) and Magnevist contrast medium $(0.1 \mathrm{mmol} / \mathrm{kg}$ body weight; Schering AG).

MW ablation device. The 2,450-MHz MW system (KY-2000; Nanjing Kangyou Medical Treatment Equipment Co., Ltd., Nanjing, China) consists of three independent MW generators, three flexible coaxial cables and three water-pumping
Table I. Clinical features of the patients and lesions.

\begin{tabular}{lc}
\hline Characteristics & Value \\
\hline Gender, n (\%) & \\
Male & $14(37.8)$ \\
Female & $23(62.2)$ \\
Mean age \pm SD, years & $43.6 \pm 10.6$ \\
Total no. of tumors & 44 \\
Mean tumor diameter \pm SD, mm & $33.0 \pm 19.1$ \\
Ablated masses, n (\%) & \\
1 & $31(83.8)$ \\
2 & $5(13.5)$ \\
3 & $1(2.7)$ \\
Diagnosis, n (\%) & \\
Hemangioma & $14(37.8)$ \\
Focal nodular hyperplasia & $5(13.5)$ \\
Inflammatory pseudotumor & $6(16.2)$ \\
Solitary necrotic nodules & $5(13.5)$ \\
Adenoma & $3(8.1)$ \\
Angiomyolipoma & $3(8.1)$ \\
Epithelioid hemangioendothelioma & $1(2.7)$ \\
Mean ablation time \pm SD, sec & $1039.9 \pm 706.2$ \\
Mean ablation energy \pm SD, kJ & $55.0 \pm 41.6$ \\
Average ablation sessions \pm SD, n & $1.3 \pm 0.5$ \\
Average ablation insertions $\pm \mathrm{SD}, \mathrm{n}$ & $2.4 \pm 1.5$ \\
Median follow-up time (range), months & \\
\hline & \\
& \\
&
\end{tabular}

SD, standard deviation.

machines, which can drive three 15-gauge cooled-shaft antennae (11-mm active tip) simultaneously. The $915-\mathrm{MHz}$ MW system (KY-2001; Nanjing Kangyou Medical Treatment Equipment Co., Ltd.) consists of two independent MW generators, two flexible coaxial cables and two water-pumping machines, which can drive two 15-gauge cooled-shaft antennae (22-mm active tip) simultaneously. The two MW generators are capable of producing 1-100 W of power output. The cooled-shaft antennae are coated with Teflon on the surface of the shaft for anti-adhesion purposes. Inside each antenna shaft, there are dual tiny channels through which distilled water or saline is circulated by a peristaltic pump, continuously cooling the shaft to prevent overheating. A thermal monitoring system attached to the MW ablation system can be used to monitor the real-time temperature during ablation.

MW ablation procedures. Lesions for MW ablation were decided upon according to the diagnoses of the nodules on contrast-enhanced imaging prior to treatment. For the nodules of indeterminate diagnosis or malignant potential, the ablated lesion included the tumor and adjacent 5-10 $\mathrm{mm}$ of normal liver tissue, particularly for those patients with chronic hepatitis, liver cirrhosis or a malignant tumor history. For the nodules of a benign nature on imaging, the ablated area was commonly 

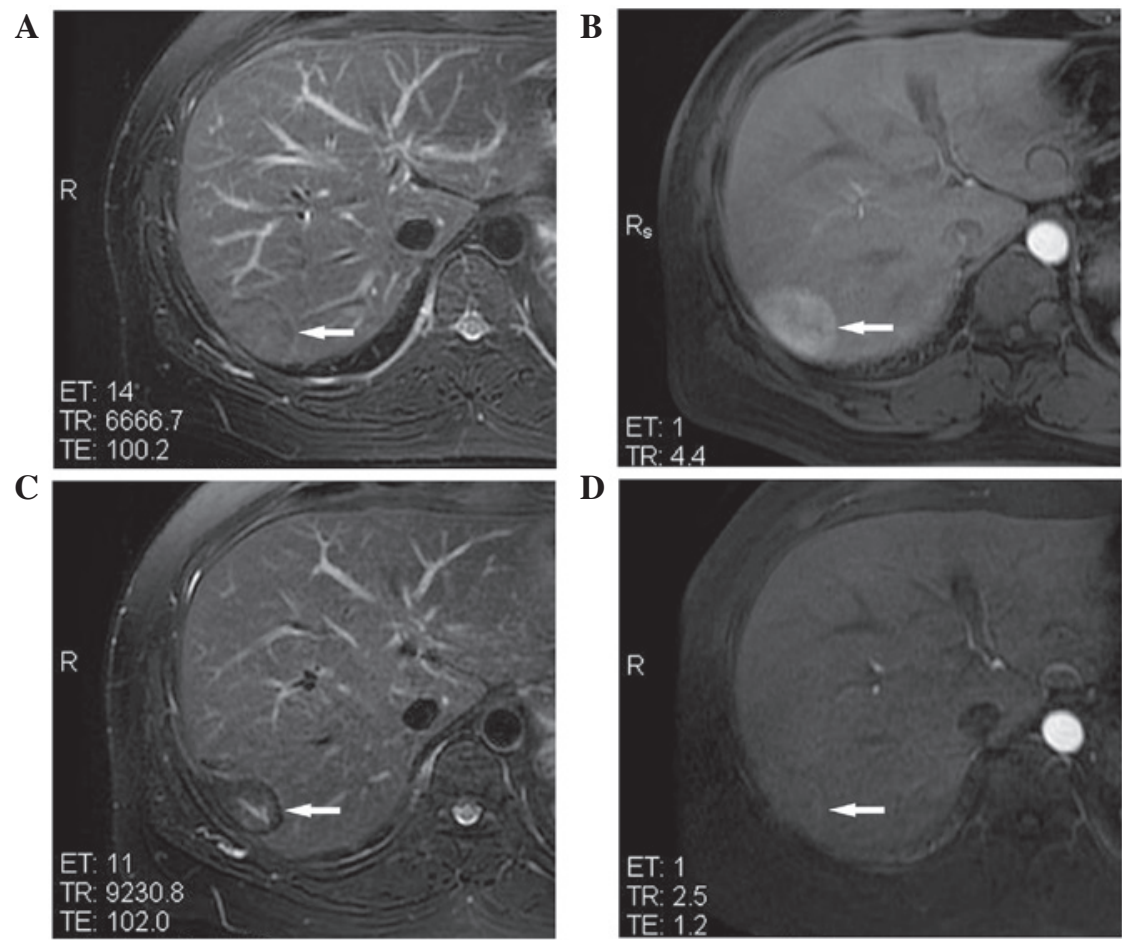

Figure 1. MRI of an inflammatory pseudotumor of the liver, with a maximal diameter of $45 \mathrm{~mm}$, in hepatic segment VII of a 38 -year-old male patient prior to and following microwave ablation. (A) T2WI showing inflammatory pseudotumor of the liver isointensity (white arrow) prior to treatment. (B) Contrast-enhanced MRI showing IPL hyperintensity (white arrow) in the arterial phase prior to treatment. (C) T2WI showing ablated lesion hypointensity (white arrow) at 1 year post-treatment. (D) Contrast-enhanced MRI showing the lack of enhancement of the ablated lesion (white arrow) in the arterial phase at 1 year post-treatment. MRI, magnetic resonance imaging; WI, weighted imaging.

recommended to cover the entire nodule only in order to minimize the damage to the normal liver tissues. When the nodule was adjacent to vital organs or structures, such as the gastrointestinal tract, major bile ducts and gallbladder, a partial ablation of the nodular volume was adopted in order to avoid injuring the vital organs by overheating, whilst still decreasing the nodular volume.

Biopsy was routinely performed prior to ablation during the procedure in order to minimize the risk of bleeding and tumor seeding. MW ablation was monitored on US in real-time, and the treatment session would be ended once the hyperechoic area induced by ablation covered the target region. When the antenna was withdrawn, the needle track was routinely cauterized to minimize the risk of bleeding and tumor seeding. If necessary, a compensatory treatment session was usually performed within 3 to 7 days depending on the volume of the residual nodule and the recovery status of the patient.

All treatments were performed in the Chinese People's Liberation Army General Hospital under intravenous anesthesia (propofol and ketamine). All procedures were performed by two experienced doctors, who each had $>10$ years of experience in the US-guided percutaneous MW ablation of liver tumors. Detailed pre-procedure plans, including the number of the antennae required, the power output setting, the emission time and the best approach to the target, were determined for each nodule on an individual basis. To decrease the number of needle insertions (10), when the maximum diameter of the nodule was $\leq 5 \mathrm{~cm}, 2,450-\mathrm{MHz} \mathrm{MW}$ ablation with a power output of $50 \mathrm{~W}$ was used, and $915-\mathrm{MHz} \mathrm{MW}$ ablation with a power output of $60 \mathrm{~W}$ was selected for nodules with a maximum diameter of $>5 \mathrm{~cm}$. If the target nodule was adjacent to any vital organs, including the major bile ducts, gallbladder, stomach and intestinal tract, one or two thermocouple needles connected to the MW ablation system would be introduced into the tissue between the nodule and the vital organ under US guidance, and temperature in real-time was monitored during ablation to avoid overheating.

Follow-up. The follow-up period was calculated starting from the beginning of the MW ablation procedures in all patients. Contrast-enhanced US, CT or MRI were repeated at 1, 3, 6, 9 and 12 months in the first year, and then at 6 to 12-month intervals after 1 year. The median follow-up period was 27.0 months (range, 6-73 months).

Statistical analysis. Data analysis was performed using SPSS 11.0 for windows (SPSS Inc., Chicago, IL, USA) and the continuous data were expressed as the mean \pm standard deviation.

\section{Results}

Outcomes of MW ablation. All 44 nodules were successfully treated. The mean numbers of ablation sessions and needle insertions were $1.3 \pm 0.5$ (range, 1-2) and 2.4 \pm 1.5 (range, 1-6) per nodule, respectively. The mean MW energy and emission time were $55.0 \pm 41.6 \mathrm{~kJ}$ (range, 13.5-207 kJ) and 1,039.9 $\pm 706.2 \mathrm{sec}$ (range, 270-3,450 sec) per nodule, respectively.

A total of 38 nodules $(86.4 \%)$ with a maximum diameter of $\leq 5 \mathrm{~cm}$ and 2 nodules $(4.5 \%)$ with a maximum diameter of 

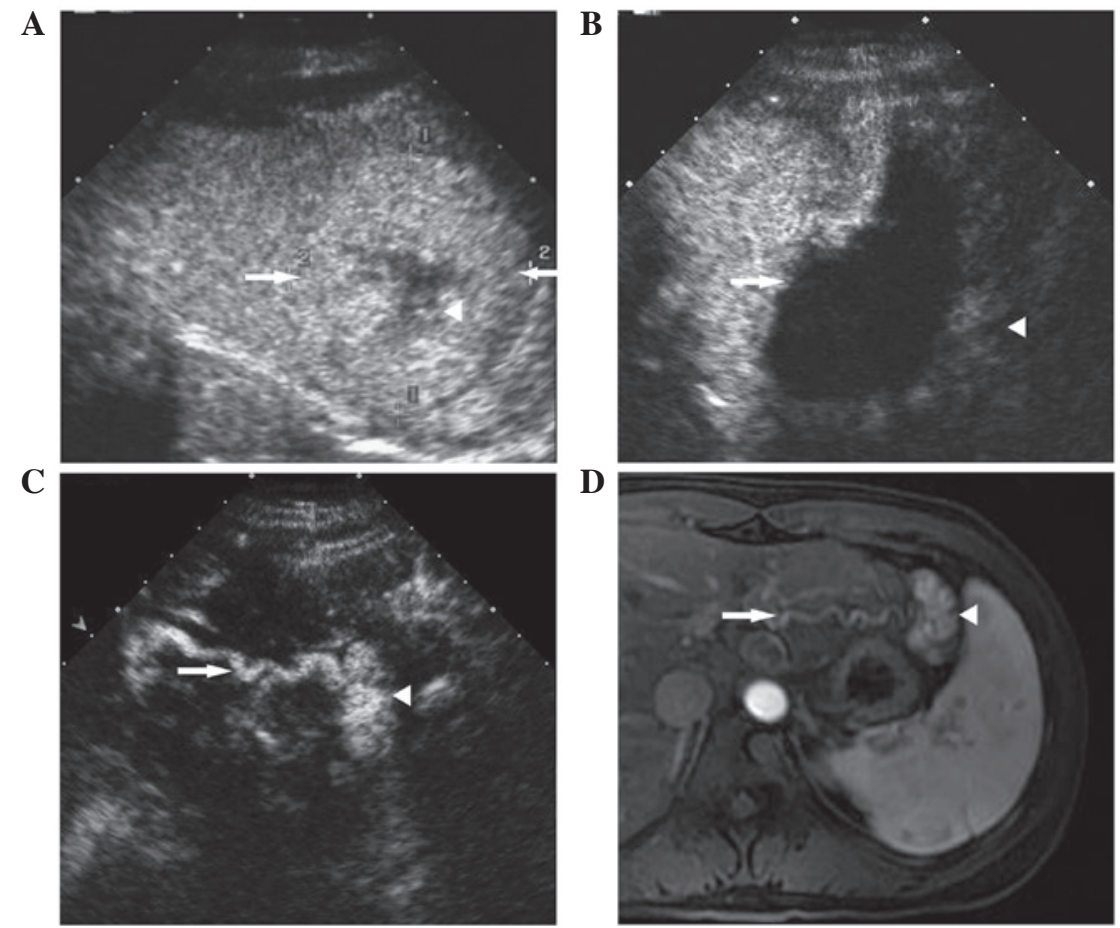

Figure 2. Contrast-enhanced US and MRI of an FNH with a maximal diameter of $56 \mathrm{~mm}$, located adjacent to the stomach in hepatic segment II of a 25-year-old female patient prior to and following microwave ablation. (A) Contrast-enhanced US showing hyperenhancement of the FNH (white arrows) and hypoenhancement of the central scar (white arrowhead) in the portal phase prior to treatment. (B) Contrast-enhanced US showing the lack of enhancement of the ablated lesion (white arrow) in the arterial phase at 4 months post-treatment, and hyperenhancement of the residual nodular area adjacent to the stomach (white arrowhead). (C) Contrast-enhanced US showing regrowth of the residual nodular area (white arrowhead), and a tortuous artery (white arrow) that occurred below the ablated lesion in the arterial phase at 1 year post-treatment. (D) Contrast-enhanced MRI showing the tortuous artery (white arrow) and hyperenhancement of the residual nodular area (white arrowhead) in the arterial phase at 1 year post-treatment. US, ultrasound; MRI, magnetic resonance imaging; FNH, focal nodular hyperplasia.

$>5 \mathrm{~cm}$ were completely ablated, with no evidence of recurrence of the peripheral ablated lesions on contrast-enhanced imaging during the follow-up period. The imaging data of 1 representative patient prior to and following treatment is shown in Fig. 1. The 3 large HCHs $(6.8 \%)$ were partially ablated due to their adjacency to the gastrointestinal tract or the vital structure of the hilum. The ablated volumes were all $>80 \%$ of the pre-procedure nodule volumes at the 1-month follow-up, and the residual areas of the nodules showed no evident progress or grew extremely slowly in the follow-up period. An FNH (2.3\%) in hepatic segment II, with a maximal diameter of $56 \mathrm{~mm}$, was partially ablated due to its adjacency with the stomach. However, the residue of the FNH continued to grow during 4 months of follow-up, as a new tortuous arterial vessel arose (Fig. 2). The patient was retreated by TAE 18 months after ablation due to the tumor regrowth.

Following ablation, the symptoms associated with the nodules in 5 patients were completely recovered or alleviated to different degrees, and the psychological pressures due to the finding of a hepatic neoplasm pre-procedure in 12 cases had been relieved.

Complications. Major complications, including abscesses, major bile duct injury, perforation of the gastrointestinal tract or gallbladder, and hemorrhage requiring embolization or surgery, did not occur in the peri-procedure and follow-up periods. Minor complications were similar to presentations recorded after MW ablation of liver cancers. The body temperature of
28 cases (75.7\%) rose to between 37.2 and $38.5^{\circ} \mathrm{C}$, lasting for 1-2 days. Management of this increase was not required in the majority of patients, and medicine to decrease the temperature was prescribed in only 4 cases. The body temperature of 2 patients reached $>38.5^{\circ} \mathrm{C}$ and was relieved by a prescription of acetaminophen $(0.64 \mathrm{~g})$ for 2 days. According to the National Cancer Institute common toxicity criteria for reporting pain (11), local pain at grade 1 was experienced in 56.8\% (21/37) of all cases, lasting 1-3 days without analgesic administration. As the ablated lesions were close to the surface of the liver, 4 patients experienced local pain at grade 2 , which was gradually relieved by oral oxycodone $(10 \mathrm{mg})$ for 3 days. The liver function tests showed that serum aspartate aminotransferase and alanine aminotransferase levels were increased in $86.5 \%$ (32/37) of all cases. The incremental levels varied, but the highest was just less than three times above the baseline value. The levels of these two enzymes were normalized in 1 month of follow-up. No marked changes were observed in other serum elements associated with liver and renal function, such as total bilirubin, direct bilirubin and creatinine. Hemoglobinuria was found at the first urination after ablation in 2 patients with large $\mathrm{HCHs}$. However, the color of the urine gradually normalized without treatment.

\section{Discussion}

For the majority of patients with BFLL, surgical resection has been considered the preferred choice of treatment (12). 
However, certain patients are non-surgical candidates or refuse surgery due to comorbidities and cosmetic requirements. As non-surgical treatments, $\operatorname{TAE}(5,13)$ and RF ablation $(6,7)$ have been advocated as minimally invasive alternatives for patients with BFLL. It is generally acknowledged that compared with RF ablation, MW ablation has similarities with regard to the rate of complete ablation, local tumor control, complications associated with treatment and the long-term survival rate when treating liver malignancies (14). Therefore, MW ablation could be a novel minimally invasive alternative for the treatment of BFLLs in patients who are non-surgical candidates.

It is well known that the most important therapeutic target in BFLLs is the minimization of the risk of rupture due to the enlargement of tumor size $(15,16)$, particularly when the nodules are located on the surface of the liver or show extrahepatic growth. Additionally, large nodules may be adjacent to the vital structures or organs such as the major bile ducts, gallbladder and gastrointestinal tract. In the present study, besides the use of thermocouple needles to monitor real-time temperature following ablation, partial ablation to achieve cytoreduction was employed to large nodules in order to minimize the nodular size and avoid major complications. The 3 large $\mathrm{HCHs}$ that were ablated partially showed acceptable results during the follow-up period, and the residual tissues exhibited no evident progression or grew extremely slowly during the follow-up period. However, the residual tissues of the large FNH that was ablated partially continued to grow during 4 months of follow-up, as a new tortuous arterial vessel arose. The patient was retreated by TAE at 18 months post-ablation due to the tumor re-growth. Thus, the therapeutic planning of complete ablation for an FNH could be recommended in further clinical application.

The complications and side effects in the present study were consistent with the standardized terminology and reporting criteria for image-guided tumor ablation (17): A major complication was defined as an event that resulted in substantial morbidity and disability, an increased level of care, hospital admission or a markedly increased hospital stay. All other complications were considered minor. In the present study, the complications of all patients encountered were minor, including fever, local pain of grade 1-2 and abnormal liver function test results. The incidence rates were similar to those previously recorded after the MW ablation of liver cancers (18). Hemoglobinuria was found after therapy in 2 cases with large $\mathrm{HCHs}$, but tended towards rapid improvement without additional remedy or management. The reason for the occurrence of hemoglobinuria may be that a mass of blood cells was damaged in an $\mathrm{HCH}$ ablation procedure.

For the treatment of BFLLs, conservative management techniques, such as regular observation, have been commonly recommended (19), and surgical resection has been used cautiously. Although MW ablation is a minimally invasive therapy compared with open hepatectomy, the same serious attitude should be advocated and the five inclusion criteria that were used in the present study should be strictly adhered to in the clinical application of US-guided percutaneous MW ablation for the treatment of BFLLs. All patients in the present study matched at least one of the five inclusion criteria. In total, 18 patients matched the first inclusion criterion and their BFLLs could not be determinately diagnosed prior to being pathologically proven. The possible reasons behind being unable to form a clear diagnosis may be as follows: It was difficult to determinately diagnose atypical and small liver neoplasms, particularly in those patients with a history of chronic hepatitis, liver cirrhosis or malignant tumors during regular follow-up. Also, certain liver neoplasms, such as ILP, HA and HEHE (20), were not easy to identify from malignancies based only on their presentations on imaging. In the present study, the HEHE in 1 patient with a history of breast carcinoma was misdiagnosed as hepatic metastasis. The correct diagnosis was confirmed by biopsy under US guidance, and no local progression or distance metastasis was reported in 6 months of follow-up after ablation.

In the treatments of large liver cancers, the application of 915-MHz MW could significantly reduce the required insertion number compared with the use of 2,450-MHz MW (10), and fewer insertions would therefore increase the safety of the therapy and decrease the risk of complications such as bleeding. In the present study, for the BFLLs with a maximum diameter of $>5 \mathrm{~cm}$, the $915-\mathrm{MHz} \mathrm{MW}$ ablation system was applied to decrease the number of insertions required. The mean number of needle insertions was $2.4 \pm 1.5$ (range, $1-6$ ) per nodule. Massive hemorrhage requiring embolization or surgery did not occur peri-procedure and blood transfusion was not necessary for any case subsequent to ablation. Furthermore, the needle tracks were routinely cauterized when the antennae were withdrawn.

In the present study, $40(90.9 \%)$ BFLLs underwent a complete ablation and the remaining $4(9.1 \%)$ nodules were partially ablated. The mean MW energy and emission time were $55.0 \pm 41.6 \mathrm{~kJ}$ (range, 13.5-207 kJ) and 1,039.9 $\pm 706.2 \mathrm{sec}$ (range, 270-3,450 sec) per nodule. A longer time and higher energy level were required for the treatment of BFLLs, particularly the larger lesions. One of the possible reasons for this was that the vessels of the BFLL were not easy to destroy by thermal ablation alone, as they have a normal vascular wall structure and a good ability to dissipate heat. The combination of TAE with ablation may be a promising method to solve this problem and treat large BFLLs, as a previous meta-analysis (21) indicated that the combination of transcatheter arterial chemoembolization with local ablation was superior to monotherapy in the treatment of patients with HCC.

The present study had certain limitations. First, only 37 patients were included due to the strict inclusion criteria. The follow-up period was also relatively insufficient. Further analysis of a large-scale study with a long-term follow-up period is required. Additionally, the present study reported a retrospective clinical outcome from a single institution only, whereas a multi-center prospective study design would have been more convincing.

In conclusion, percutaneous MW ablation under US guidance is a safe, effective therapy for selected patients with BFLL and can be considered as a minimally invasive alternative to surgery. Clinically, it should be applied cautiously and the inclusion criteria mentioned in present study should be recommended.

\section{Acknowledgements}

This study was financially supported by the National Scientific Foundation Committee of China (grant nos. 30825010 and 
81430039) and the Clinical Scientific Research Grant of the Chinese People's Liberation Army General Hospital (grant no. 2012FC-ZHCG-1008).

\section{References}

1. Bartolotta TV, Midiri M, Galia M, Rollandi GA, Cademartiri F, Lagalla $\mathrm{R}$ and Cardinale AE: Characterization of benign hepatic tumors arising in fatty liver with SonoVue and pulse inversion US. Abdom Imaging 32: 84-91, 2007.

2. Petri A, Höhn J, Kókai EL, Savanya GK and Lázár G: Surgery of benign liver tumors: Indications for treatment: Twenty years experience. Hepatogastroenterology 55: 592-595, 2008.

3. Tanabe KK: The past 60 years in liver surgery. Cancer 113 (Suppl): S1888-S1896, 2008.

4. Feng ZQ, Huang ZQ, Xu LN, Liu R, Zhang AQ, Huang XQ, Zhang WZ and Dong JH: Liver resection for benign hepatic lesions: A retrospective analysis of 827 consecutive cases. World J Gastroenterol 14: 7247-7251, 2008

5. Srivastava DN, Gandhi D, Seith A, Pande GK and Sahni P: Transcatheter arterial embolization in the treatment of symptomatic cavernous hemangiomas of the liver: A prospective study. Abdom Imaging 26: 510-514, 2001

6. Cui Y, Zhou LY, Dong MK, Wang P, Ji M, Li XO, Chen CW, Liu ZP, Xu YJ and Zhang HW: Ultrasonography guided percutaneous radiofrequency ablation for hepatic cavernous hemangioma. World J Gastroenterol 9: 2132-2134, 2003.

7. Hinshaw JL, Laeseke PJ, Weber SM and Lee FT Jr: Multiple-electrode radiof requency ablation of symptomatic hepatic cavernous hemangioma. AJR Am J Roentgenol 189: W146-W149, 2007.

8. Ahmed M and Goldberg SN: Thermal ablation therapy for hepatocellular carcinoma. J Vasc Interv Radiol 13: S231-S244, 2002

9. Wright AS, Sampson LA, Warner TF, Mahvi DM and Lee FT Jr: Radiofrequency versus microwave ablation in a hepatic porcine model. Radiology 236: 132-139, 2005.

10. Liu FY, Yu XL, Liang P, Wang Y, Zhou P and Yu J: Comparison of percutaneous $915 \mathrm{MHz}$ microwave ablation and $2450 \mathrm{MHz}$ microwave ablation in large hepatocellular carcinoma. Int $\mathrm{J}$ Hyperthermia 26: 448-455, 2010.
11. National Cancer Institute: Cancer therapy evaluation program: Common Toxicity Criteria, version 2.0. http://ctep.cancer.gov/reporting/ctc.html. Accessed July 1, 2005.

12. Arnoletti JP and Brodsky J: Surgical treatment of benign hepatic mass lesions. Am Surg 65: 431-433, 1999.

13. Deutsch GS, Yeh KA, Bates WB III and Tannehill WB: Embolization for management of hepatic hemangiomas. Am Surg 67: $159-164,2001$

14. Lu MD, Xu HX, Xie XY, Yin XY, Chen JW, Kuang M, Xu ZF, Liu GJ and Zheng YL: Percutaneous microwave and radiofrequency ablation for hepatocellular carcinoma: A retrospective comparative study. J Gastroenterol 40: 1054-1060, 2005.

15. Vokaer B, Kothonidis K, Delatte P, De Cooman S, Pector JC and Liberale G: Should ruptured liver haemangioma be treated by surgery or by conservative means? A case report. Acta Chir Belg 108: 761-764, 2008.

16. AiuraK, Ohshima R, MatsumotoK, Ishii S, Arisawa Y,Nakagawa M and Noga K: Spontaneous rupture of liver hemangioma: Risk factors for rupture. J Hep Bil Pancr Surg 3: 308-312, 1996.

17. Goldberg SN, Grassi CJ, Cardella JF, Charboneau JW, Dodd GD III, Dupuy DE, Gervais DA, Gillams AR, Kane RA, Lee FT Jr, et al; Society of Interventional Radiology Technology Assessment Committee and the International Working Group on Image-guided Tumor Ablation: Image-guided tumor ablation: Standardization of terminology and reporting criteria. J Vasc Interv Radiol 20 (Suppl): S377-S390, 2009.

18. Liang P, Wang Y, Yu X and Dong B: Malignant liver tumors: Treatment with percutaneous microwave ablation-complications among cohort of 1136 patients. Radiology 251: 933-940, 2009.

19. Terkivatan T, de Wilt JH, de Man RA, van Rijn RR, Zondervan PE, Tilanus HW and IJzermans JN: Indications and long-term outcome of treatment for benign hepatic tumors: A critical appraisal. Arch Surg 136: 1033-1038, 2001.

20. Cardinal J, de Vera ME, Marsh JW, Steel JL, Geller DA, Fontes P, Nalesnik M and Gamblin TC: Treatment of hepatic epithelioid hemangioendothelioma: A single-institution experience with 25 cases. Arch Surg 144: 1035-1039, 2009.

21. Gu L, Liu H, Fan L, Lv Y, Cui Z, Luo Y, Liu Y, Li G, Li C and Ma J: Treatment outcomes of transcatheter arterial chemoembolization combined with local ablative therapy versus monotherapy in hepatocellular carcinoma: A meta-analysis. J Cancer Res Clin Oncol 140: 199-210, 2014. 